\title{
Application of Graph Theory in Online Network Services to Determine the Shortest Journey
}

\author{
B. Logeshwary Natarajan \\ Department of Humanities and Science, Rajalakshmi Institute of Technology, Chennai, 602124, INDIA. \\ Email: logeshwary.b@ ritchennai.edu.in \\ M.K. Balaji \\ Department of Humanities and Science, Rajalakshmi Institute of Technology, Chennai, 602124, INDIA. \\ Email: hod.hs@ritchennai.edu.in
}

ABSTRACT-

In this paper, we determine the shortest route in online network services by applying the concept of graph theory. The investigation shows how the journey can be visualized as graphs in the nature of vertices and edges. In our research paper, the smallest distance from starting point to final destination is estimated using google map which provides the smallest cumulative cost. In this paper, our focus is to achieve the process in working of google map services using graph theoretical approach to determine the shortest journey and its implementation in computer science using Floyd Warshall algorithm. In this paper, we also give illustrations and prove some results.

Keywords - Distance graph, Online network services, Shortest route problem, Floyd Warshall algorithm

Date of Submission: Nov 23, 2018

Date of Acceptance: Feb 10, 2019

\section{INTRODUCTION}

$T_{\text {he origin of graph theory started with the problem of }}$ Konigsberg bridge in 1736 [8], [17]. This problem lead to the concept of Eulerian Graph. Euler studied the problem of Konigsberg bridge and constructed a structure to solve the problem called Eulerian graph. In 1840, A.F Mobius gave the idea of complete graph and bipartite graph and Kuratowski proved they are planar by means of recreational problems. The concept of tree was implemented by Gustav Kirchhoff in 1845 and illustrated graph theoretical ideas in the calculation of currents in electrical networks or circuits. In 1852, Thomas Gutherie found the famous four colour problem. In 1856, Thomas P. Kirkman and William R. Hamilton studied cycles on polyhydra and invented the concept called Hamiltonian graph. In 1913, H. Dudeney mentioned a puzzle problem. Although the four colour problem was invented it was solved only after a century by Kenneth Appel and Wolfgang Haken. This fertile period is considered as the birth of Graph Theory [9],[10].

Graphs are essential because graph is a key to express information in pictorial form. Most of the problems which are considered hard to determine can be easily solved using graph theory. Proper understanding of different graphs and its techniques are required to solve the conflicts in the real world in the process of obtaining the solution. Graph theory is applied in the various fields like computer science, Biology, Chemistry and Operation Research [12].

\section{GRAPH TheORY AND SHORTEST ROUTE Problem}

A Graph $G$ consists of pair $(\mathrm{V}(\mathrm{G}), \mathrm{E}(\mathrm{G}))$ where $\mathrm{V}(\mathrm{G})$ is a non-empty finite set whose elements are vertices and $E(G)$ is a set of unordered pairs of distinct elements of $V(G)$.
The number of vertices in a graph is called the order and number of edges in a graph is called the size. The degree of a vertex of a graph is the number of edges incident to the vertex with loop counted twice. The degree of vertex denoted by $\operatorname{deg}(\mathrm{v})$ [9]. The maximum degree of a graph $\mathrm{G}$ denoted by $\Delta(\mathrm{G})$ and the minimum degree of graph $\mathrm{G}$ denoted by $d(G)$. An undirected graph is one in which edges have no orientation. The edge $(a, b)$ is identical to the edge (b,a). A directed graph in which each edge is represented by an ordered pair of two vertices. $(\mathrm{Vi}, \mathrm{Vj})$ denotes an edge from $\mathrm{Vi}$ to $\mathrm{Vj}$ from first vertex to second vertex. A graph $\mathrm{G}=(\mathrm{V}, \mathrm{E})$ with no loops and no multiple edges or parallel edges is called simple graph. In a graph $\mathrm{G}=(\mathrm{V}, \mathrm{E})$ if a pair of vertices are allowed to join by more than one edges those edges are called parallel edges and the resulting graph is called multi graph. Edges drown from a vertex to itself is called a loop. In a graph $\mathrm{G}=(\mathrm{V}, \mathrm{E})$ two vertices are said to be adjacent if there exists an edge between the two vertices. A graph is said to be acyclic graph if it has no cycles. An acyclic graph which is connected is called a tree and if one or more of the edges disconnected then the acyclic graph is a called a forest. [17].

In graph theory, the problem of finding a route between two vertices in a graph such that the sum of its weights of its constituent edges is minimized is called as the shortest route problem [3]. The Shortest route problem is a fundamental problem in network analysis. Graph is a pictorial representation of shortest route problem which contains set of vertices and edges. Vertices are called as nodes. Edges connect the pair of vertices. It is possible to walk along the edges by moving from one vertex to another vertices depending on whether one can walk along the edges by both sides or by only one side according as the graph is directed graph or undirected graph. The 
lengths of the edges are called weights for estimating the smallest route from source to the destination [13].

\section{GRAPH THEORY IN GOOGLE MAP SERVICES}

A Map is a pictorial representation of a region represented on a flat surface. The effort of a map illustrates detailed features of a geographical area like boundaries, roads, physical features, topography, climates, and natural is time resources [1],[2]. The problem is explained manually which is time consuming and this difficulty can be easily resolved by using mathematical tools like shortest route algorithms [2]. Google maps helps in achieving the destination from one place to other. Graph theory is the study of points and lines where set of points are called vertices. Lines connecting points are called edges. Graphs can be either directed or undirected depending upon the walk on one side or both sides. The lengths of the edges are called weights from one intersection to other which is the cost for optimization used to evaluate the smallest route from source to the destination. Google map identifies all possible routes from the starting point to the ending point. The shortest and quickest route will be displayed by the server. Figure 1 shows all possible routes between Avadi and Ambattur along the distance in kilometers. Here three possible routes are sorted out by the google map out of which the fastest route is highlighted in blue with distance $9.4 \mathrm{~km}$ in 23 minutes [5].

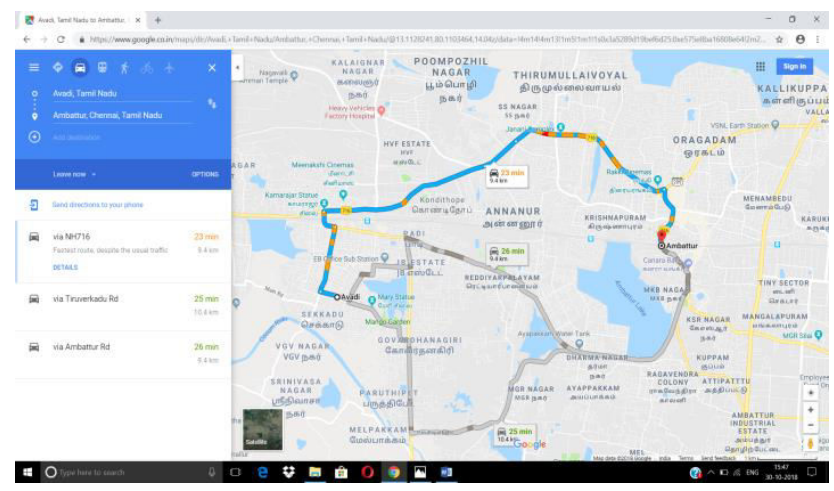

Figure 1. Google map

To illustrate the application of graph theory in google map services let us consider the real time situation for analysis. Assume the salesman in a hurry to drive from $\mathrm{L}_{4}$ lane to $4^{\text {th }}$ avenue to meet a very important client. He wants to use the route that needs the least travel time. The graph shows the time she needs to spend on driving from place to place between the source and the destination. Estimate the shortest route for the salesman from $4^{\text {th }}$ avenue to $\mathrm{L}_{4}$ lane [14],[15].

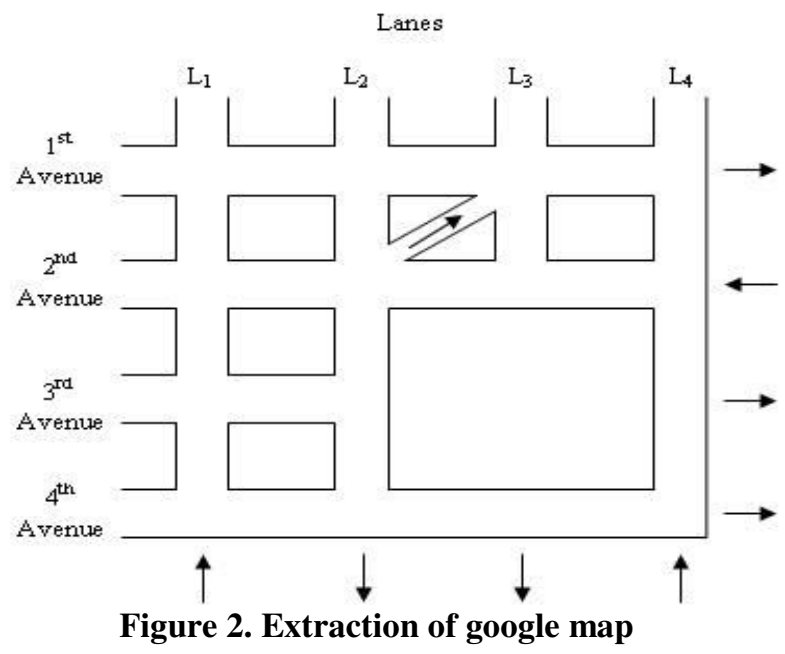

Extract the graph from the google map. The directions of avenues and lanes are $1^{\text {st }}, 3 \mathrm{rd}, 4$ th Avenue are moving to right. $2^{\text {nd }}$ Avenue is moving to left whereas the lanes $\mathrm{A}$ and $\mathrm{D}$ are moving to up but lanes $\mathrm{B}$ and $\mathrm{C}$ are moving to down. The weights in terms of cost are denoted on edges from one intersection to other. Plot the points where the lines meet at intersection as vertices. Label the vertices as A, B, C, D, E, F, G, H, I, J, K, L and M. Connect the vertices with edges that leads to destination as per the direction of the google map. Mark the directions mentioned in the google map for avenues and lanes [16].

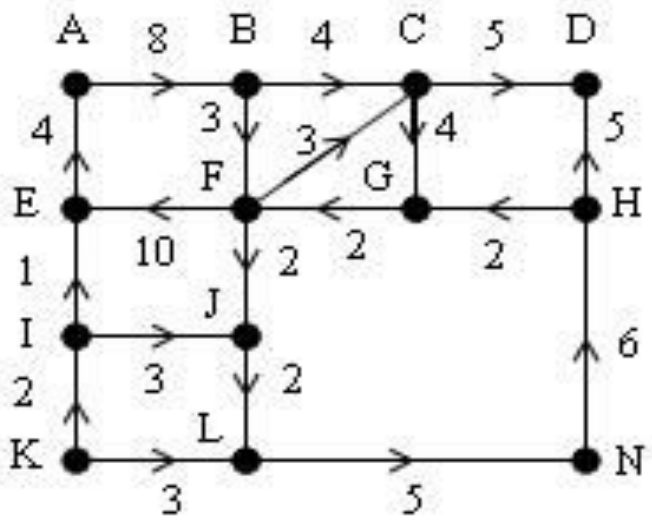

Figure 3. Distance graph G

The weights of the graphs differ due to parameters like speed limits, road work distance. Latest updates on traffic data and various datas are sent to server and these can be utilized in calculating quickest way from one place to other. The cumulative sum of the routes will give the optimum cost. Identify all the possible routes for the salesman from $\mathrm{L}_{4}$ lane to $4^{\text {th }}$ avenue to reach the destination. Here there are two ways to reach $\mathrm{L}_{4}$ lane from $4^{\text {th }}$ avenue. Here the first route direction is from $\mathrm{K} \rightarrow \mathrm{I} \rightarrow \mathrm{E} \rightarrow \mathrm{A} \rightarrow \mathrm{B} \rightarrow \mathrm{C} \rightarrow \mathrm{D}$ and second route direction is from $\mathrm{K} \rightarrow \mathrm{L} \rightarrow \mathrm{N} \rightarrow \mathrm{H} \rightarrow \mathrm{D}$. The cumulative sum of first route direction is given by $\mathrm{K}+\mathrm{I}+\mathrm{E}+\mathrm{A}+\mathrm{B}+\mathrm{C}+\mathrm{D}=24$ and the cumulative sum of second route direction is given by $\mathrm{K}+\mathrm{L}+\mathrm{N}+\mathrm{H}+\mathrm{D}=19$. Here the second route is the shortest route which is the optimum [2],[5]. 


\section{GRAPH THEORY IN ONLINE NETWORK SERVICES}

OLA cabs an online transportation network company founded by ANI Technologies developed into a network of more than 1,000,000 vehicles across 169 cities. The cabs are booked through a mobile application or website consisting of more than 150,000 bookings per day in India with 6000 employees in the year 2018. The essential fact for critical functioning of OLA is not only to find the location but also the skill to match drivers with users in a potential manner. The problem starts with locations. The backend operations of OLA processes several millions of user demands and transferring each of the need to one or more or all of the closest drivers. The major work is to assign the right driver for the right drive in addition to process the incoming demand and finding the location based on operator coordinates and drivers with closest coordinates. In this scenario, there are two different cabs reaching the user based on users request. Here our aim is to calculate the minimum required distance for the cab to reach the user.[15]

To illustrate this application in online network OLA let us consider the figure 2 for analysis. Estimate the shortest route for the user from $4^{\text {th }}$ avenue to $L_{1}$ lane [14]. Repeat the process as explained for the google map services in section III. Here there are two ways to reach $\mathrm{L}_{4}$ lane from $4^{\text {th }}$ avenue. Here the first route is from $\mathrm{K} \rightarrow \mathrm{I} \rightarrow \mathrm{E} \rightarrow \mathrm{A}$ and second route is $\mathrm{K} \rightarrow \mathrm{L} \rightarrow \mathrm{N} \rightarrow \mathrm{H} \rightarrow \mathrm{G} \rightarrow \mathrm{F} \rightarrow \mathrm{E} \rightarrow \mathrm{A}$. The cumulative sum of first route direction is $\mathrm{K}+\mathrm{I}+\mathrm{E}+\mathrm{A}=7$ and the cumulative sum of second route direction is $\mathrm{K}+\mathrm{L}+\mathrm{N}+\mathrm{H}+\mathrm{G}+\mathrm{F}+\mathrm{E}+\mathrm{A}=32$. Here the first route is the shortest route which is the optimum cost. [2],[5][10].

\section{GRAPH THEORETICAL APPROACH TO DETERMINE SHORTEST JOURNEY AND SOME RESULTS}

In this paper, we describe a new graph theoretical method to determine the shortest route. Here we consider the figure 3 to find the minimum cost path from vertex $\mathrm{K}$ to vertex $\mathrm{D}$ by graph theoretical approach. There are various options to travel from vertex $\mathrm{K}$ to vertex $\mathrm{D}$ but our aim is to find the shortest path with a minimum transportation cost. In this paper our motive is to achieve the minimal acyclic graph for the given graph. [2],[10].

The following steps describes the determination of shortest path.

Step 1: Plot only the vertices of the graph without edges and loops.

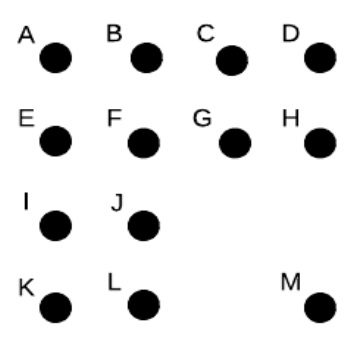

Figure 4. Complement of $\mathbf{G}\left(G^{c}\right)$

Step 2: Draw the edges of the graph from the lowest cost vertex with value 1 without loops, parallel edges and cycles from vertex $\mathrm{K}$ to vertex $\mathrm{D}$.

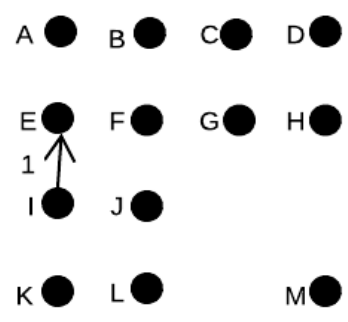

Figure 5

Step 3: Repeat the process by including higher value edges one by one from the lowest value until the highest cost vertex with value 8 is drawn by removing cycles and loops. After repeated iteration we arrive at the acyclic graphs which are devoid of cycles.

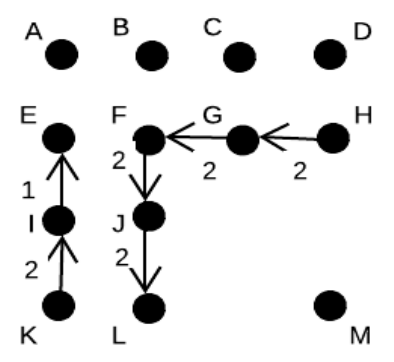

Figure 6

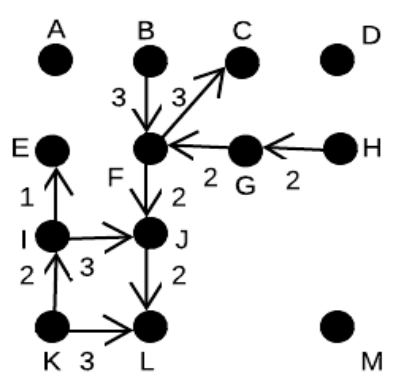

Figure 7 


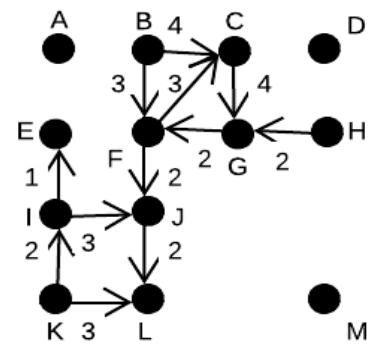

Figure 8

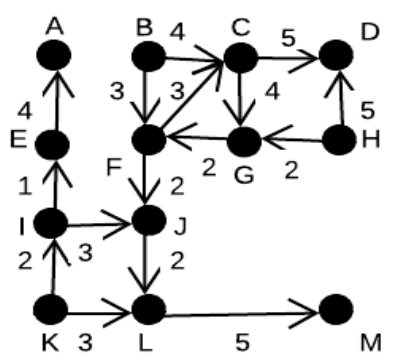

Figure 9

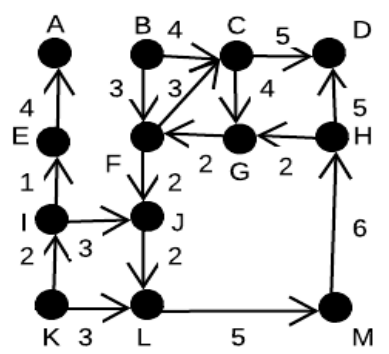

Figure 10

Step 4: The graph thus formed is a minimal acyclic graph. Here there are two ways to reach $\mathrm{L}_{4}$ lane from $4^{\text {th }}$ avenue. Here the first route direction is from $\mathrm{K} \rightarrow \mathrm{I} \rightarrow \mathrm{E} \rightarrow \mathrm{A} \rightarrow \mathrm{B} \rightarrow \mathrm{C} \rightarrow \mathrm{D}$ and second route direction is from $\mathrm{K} \rightarrow \mathrm{L} \rightarrow \mathrm{N} \rightarrow \mathrm{H} \rightarrow \mathrm{D}$. The cumulative sum of first route direction is given by $\mathrm{K}+\mathrm{I}+\mathrm{E}+\mathrm{A}+\mathrm{B}+\mathrm{C}+\mathrm{D}=24$ and the cumulative sum of second route direction is given by $\mathrm{K}+\mathrm{L}+\mathrm{N}+\mathrm{H}+\mathrm{D}=19$. Here the second route is the shortest route which is the optimum.

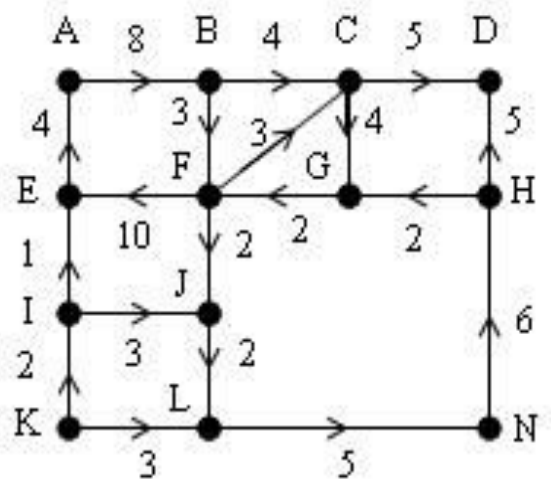

Figure 11. G*

\section{SOME RESUlts AND ObSERVATIONS}

Theorem: If $\mathrm{G}^{*}$ is any spanning acyclic graph of $\mathrm{G}$, then then each circuit of $\mathrm{G}$ connects the complement $\mathrm{G}^{\mathrm{I}}$ by an edge.

Proof: Consider the spanning acyclic graph $\mathrm{G}^{*}$. Let $\mathrm{C}^{*}$ be the cutset whose removal splits a components of $\mathrm{G}$ in to subgraphs If we add to $G^{*}$ any edge of $G$ not contained in $\mathrm{G}^{*}$ we obtain a cycle. The collection of cycles formed in this way by adding separately each edge of $G$ not contained in $\mathrm{G}^{*}$ which is a contradiction. That is, a cycle of $\mathrm{G}$ having no edge in common with the complement of $\mathrm{G}^{*}$ is contained in $\mathrm{G}$. Hence proved the theorem.

Corollary: Let $\mathrm{C}^{*}$ be a collection of edges a graph $\mathrm{G}$. If $\mathrm{C}^{*}$ has a common edge with $\mathrm{G}$, then $\mathrm{C}^{*}$ contains a cutset.

\section{IMPLEMENTATION OF GRAPH THEORETICAL APProACH To DETERMINE SHORTEST JOURNEY IN COMPUTER SCIENCE USING FLOYD WARSHALL ALGORITHM}

Floyd Warshall algorithm is viewed as all pair shortest path algorithm. In our research, we explain the graph theoretical approach to determine the shortest journey between any two nodes. In our research, we implement the process using the programming tool called Floyd Warshall algorithm to find the shortest route in a weighted graph which in turn is very helpful to find all possible routes in the graph between every pair of vertices. This algorithm calculates the value of the shortest journey between each pair of vertices in a graph. A single execution generates all the possible routes between every pair of vertices. The time complexity of Floyd Warshall algorithm is $\mathrm{O}\left(\mathrm{V}^{3}\right)$ time as three nested for loops are used to solve the distance matrix. Here we mention the pseudocode for the Floyd Warshall algorithm.[10].

\section{Pseudocode:}

if $\mathrm{u}==\mathrm{v}$ :

$\mathrm{M}[\mathrm{u}][\mathrm{v}]=0$

if there is a edge from $u$ to $v$ :

$\mathrm{M}[\mathrm{u}][\mathrm{v}]=$ weight $(\mathrm{u}, \mathrm{v})$

else:

$\mathrm{M}[\mathrm{u}][\mathrm{v}]=$ infinity

for $\mathrm{u}=1$ to $\mathrm{N}$

for $\mathrm{v}=1$ to $\mathrm{N}$

for $\mathrm{w}=1$ to $\mathrm{N}$

$\operatorname{dist}[\mathrm{w}][\mathrm{u}][\mathrm{v}]=\min (\operatorname{dist}[\mathrm{w}-1][\mathrm{u}][\mathrm{v}], \operatorname{dist}[\mathrm{w}-1][\mathrm{u}][\mathrm{w}]+\operatorname{dist}[\mathrm{w}-$ $1][w][v])$.

\section{CONCLUSION}

In this paper, we provide clear idea to determine the shortest journey using google map services and online transportation network with the application of graph theory. The shortest route problem in graph theory determines the smallest and quickest route from the source 
to destination. In our research paper, we describe the new graph theoretic method to estimate minimum cost path and prove some results. Thus it is amply clear that the power of computer must be combined with the ingenuity of mathematical techniques to enhance the manipulation speed hence we implement the process using Floyd Warshall Algorithm to find the all possible routes in distance graph.

\section{REFERENCES}

[1] Akinwol Agnes Kikelomo, Yekini Nureni Asafe, Adelokun Paul and N. Lawal Olawale, Design and Implementation of Mobile Map Application for Finding Shortest Direction between Two Pair Locations Using Shortest Path Algorithm: A Case Study, Int. J. Advanced Networking and Applications, 9(1), 2017, 3300-3305.

[2] K.S. Avdhesh, and K. Sourabh, Finding of Shortest Path from Source to Destination by traversing every node in wired Network, International Journal of Engineering and Technology, 5, 2013, 2655-2656.

[3] N.L. Biggs, Algebraic Graph Theory, (Cambridge University Press, Cambridge, 1993).

[4] A.K. Bincy, and B. Jeba Presitha, Graph Coloring and its Real Time Applications an Overview, International Journal of Mathematics and its Applications, 5(4), 2017, 845-849.

[5] B. Bollobas, (1990), Graph Theory: An Introductory Course, (Springer, New York , 1990), 3rd corrected edition.

[6] Cristina Dalfo and Miquel Angel Fiol, Graphs, friends and acquaintances, Electronic Journal of Graph Theory and Applications, 6(2), 2018, 282-305.

[7] R. Diestel, Graph Theory, (Springer, New York, 1997).

[8] C.D. Godsil, and G. Royle, G., Algebraic Graph Theory, (Springer-Verlag, New York, 2001).

[9] F. Harary, F., Graph Theory, (Addison-Wesley, Reading, MA, 1969).

[10] J.L. Hyun, and D.K. Soo, A service-based approach to developing Android Mobile Internet Device (MID) applications, Service-Oriented Computing and Applications, 2010.

[11] H. Mojo, Graph theory (2016). Available at www.en.m.wikipedia.org/wiki/Graph_Theoy.

[12] Narasingh Deo, Graph theory with applications to engineering and computer science, (Prentice Hall of India, 1990).

[13] Pranav Patel and Chirag Patel, Various graphs and their applications in real world, International Journal of Engineering Research \& Technology (IJERT), 2(12), 2013.

[14] S.G. Shirinivas, S. Vetrivel, and N.M. Elango, Application of Graph theory in Computer Science an overview, International Journal of Engineering Science and Technology, 2010.

[15] S. Venu Madhava Sarma, Applications of graph theory in human life, International Journal of Computer Application, 1(2), 2012.
[16] D.B. West, Introduction to Graph Theory, (PrenticeHall, Englewood Cliffs, NJ, 2nd edition, 2000).

\section{Biographies and Photographs}

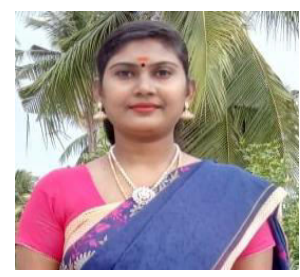

Dr. B. Logeshwary Natarajan is a Senior Assistant Professor in Department of Mathematics at Rajalakshmi Institute of Technology, Chennai, India. In the year 2005, she received her B.Sc degree and in the year 2007 she received her M.Sc degree both in Mathematics from Jaya College of Arts and Science, Chennai, India. In the year 2008, she received her M.Phil degree in Mathematics from Pachaiyappas College, Chennai, India. In the year 2017, she received her Ph.D degree in Mathematics from Bharathiar University, Coimbatore, India. She has 10 years of teaching experience. Her research interests include Algebra, Real Analysis, Engineering Mathematics and Graph theory.

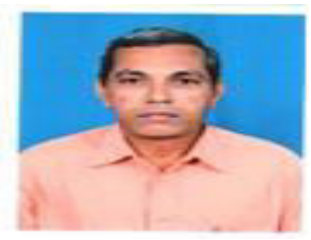

Dr. M.K. Balaji is a Professor and Head in Department of Humanities and Science at Rajalakshmi Institute of Technology, Chennai, India. In the year 1986, he received his B.Sc degree in Mathematics from Arignar Anna Government Arts College, Cheyyar, India. He has completed his M.Sc(1989) and completed his M.Phil(1990) both from Loyola College Chennai, India. In the year 2014, he received his Ph.D degree in Mathematics from Sri Chandrasekharendra Saraswathi Viswa Mahavidyalaya University, Kanchipuram, India. He has 28 years of teaching experience. His research interests include Solid geometry, Algebra, Complex Analysis and Graph theory. 